\title{
PULSAR OBSERVATIONS AND NEUTRON STAR MODELS
}

\author{
GERHARD BÖRNER* and JEFFREY M. COHEN** \\ Laboratory for High Energy Astrophysics, NASA/Goddard Space Flight Center, Greenbelt, Md., U.S.A.
}

\begin{abstract}
Information about the physical parameters of neutron stars is obtained from pulsar observations. The energy balance of the Crab Nebula and the Vela X remnant allows one to derive limits for the masses of the Crab and Vela pulsars. Glitch observations provide further clues on the masses of these two pulsars. The degree of confidence with which one should believe the derived numbers is pointed out. The possibility of observing neutron stars in binary systems as pulsating $\mathrm{X}$-ray sources is discussed. Finally, the importance of observing redshifted gamma ray lines from the surface of neutron stars, and thus directly measuring either individual or statistical properties of these objects, is pointed out.
\end{abstract}

\section{Introduction}

Besides the well-known chain of arguments that leads, by process of elimination (Maran and Cameron, 1969), to the generally accepted conclusion that pulsars are rotating neutron stars, there is another very gratifying result which stems from the comparison of neutron star models and pulsar observations. It turns out that for any reasonable equation of state the neutron star models, computed as outlined in the paper by Cohen and Börner in this volume (hereafter called Paper I), have moments of inertia which are just of the right order of magnitude to explain the observed energy input into the Crab Nebula as the loss of rotational energy from a rotating neutron star with the parameters of the Crab pulsar PSR $0531+21$. This is a major triumph of the interaction of theory and experiment in this field. It has already been mentioned in the papers of H. A. Bethe and D. Pines in this volume, but this information really belongs to the subject matter of the present paper and has therefore been pointed out again. We shall discuss in detail the energy balance of the Crab and Vela Nebulae later.

Except for what was pointed out above, the interaction between theory and observation is very slim to date; so much so that no observer is participating in this conference and observations have to be discussed by theorists, one of whom has never even looked through a telescope. But this is understandable because the wealth of pulsar observations is overwhelming, and a comprehensive understanding still has not been achieved. Only the puzzling 'glitches' of the Crab and Vela pulsar have been well incorporated into various competing theories. These will be discussed at the end of this paper.

In the following we shall attempt to gather some of the pieces in the puzzle that link neutron star models and pulsar observations. We shall try very ambitiously to derive parameters for the rotating neutron stars that are represented by the names Crab and Vela in the astronomical observations.

* Permanent address: Max-Planck-Institut für Physik und Astrophysik München, Germany and National Academy of Science-NRC Research Associate.

** Permanent address: Physics Department, University of Pennsylvania, Philadelphia, Pennsylvania 19104. National Academy of Science-NRC Senior Research Associate. 


\section{Radio Pulsars}

All of the 61 pulsars listed by Manchester and Taylor (1972), except for Crab and Vela, are seen only by their pulsed radio emission. It is quite obvious that the pattern, shape and polarization of these radio pulses contains quite a lot of information on the internal structure of the pulsar. We are, however, not yet able to understand the message we are getting. No satisfying quantitative description of the electromagnetic link between the rotating neutron star and the radiation pattern of the pulsar has been given so far. Indeed, not even the case of a magnetosphere of radiative particles, where the axis of the magnetic field coincides with the rotation axis of the neutron star, has been solved. Furthermore, to explain the pulse producing mechanism one would have to treat the much more complicated case of at least a slight deviation from axial symmetry.

In the absence of a detailed mechanism for pulsar emission, only energy considerations can be employed to obtain information on the physical properties of the rotating neutron star. For 22 of the 61 listed pulsars (Manchester and Taylor, 1972) both frequency $\Omega$ and change of frequency $\dot{\Omega} \equiv \mathrm{d} \Omega / \mathrm{d} t$ have been measured. Then by determining their rate of loss of energy $E_{\text {rot }}=I \Omega \dot{Q}$ we could in principle find the moment of inertia $I$ of these neutron stars. This in turn would precisely fix the mass and density profile of the star once an equation of state had been chosen.

The energy in radio pulses $E_{\text {puls }}$ probably is only a small part of the total energy release, and therefore only very crude limits on the physical parameters of a neutron star may be derived. Even this modest undertaking does not look very promising, however, since neither the flux in radio pulses (a well-defined mean value for a specific pulsar) nor the distance to pulsars are known to a high accuracy.

The flux in radio pulses is known to fluctuate very strongly on a scale of weeks. This makes it very difficult to define a quantity like the mean pulse intensity at a given frequency for any pulsar. Furthermore the distance to pulsars can only be estimated (again excepting Crab and Vela) by their dispersion measure, which gives the average value of the electron density along the line of sight. To derive the distance of pulsars from the dispersion measure one would need a precise knowledge of the interstellar medium. In reality, however, we use the pulsars to obtain more information on the interstellar medium and it has been found that in a region of $100 \mathrm{pc}$ around the Sun, the average electron density is almost $0.1 \mathrm{~cm}^{-3}$. This is roughly comparable to the average density of the atomic hydrogen in that region, and indicates that we are surrounded neither by a classical HII region nor by an HI region (cf., Biermann, 1972).

Thus there is virtually no feedback to the neutron star models through the observations of radio pulses. If we nevertheless make some crude estimates of the energy in radio pulses for various pulsars, we find $E_{\text {puls }} \approx 10^{28 \pm 2} \mathrm{erg} \mathrm{s}^{-1}$. While that result is laden with all the uncertainties discussed above, it permits one rather vague but still interesting conclusion: assuming moments of inertia between $10^{44}$ and $10^{45} \mathrm{~g} \mathrm{~cm}^{2}$ we can compute the rotational energy output $\left(\dot{E}_{\text {rot }}\right)$ for various pulsars. We then find that the energy in radio pulses for the Crab pulsar $\dot{E}_{\text {puls }} \approx 10^{-9} \dot{E}_{\text {rot }}$, while for many of the older pulsars $\dot{E}_{\text {puls }}$ is a much bigger fraction of $\dot{E}_{\text {rot }}$, such as PSR $0809+74$, where 
$\dot{E}_{\mathrm{pu} 1 \mathrm{~s}} \approx \dot{E}_{\mathrm{rot}}$. This seems to indicate that as pulsars grow older they spend a bigger and bigger fraction of their rotational energy output in the production of radio pulses.

\section{The Crab Pulsar}

The Crab pulsar PSR $0531+21$ is located at the center of the Crab Nebula, the remnant of a supernova that exploded in 1054. Because of its location in the nebula a distance estimate independent of the dispersion measure can be obtained and, furthermore, the energy balance of the nebula can be used to get more information on the energy output of this pulsar. In addition, pulses from this object have been observed not only in the radio regime but also in the optical and X-ray frequencies.

The supernova of 1054 is widely considered to have been of type I, but Minkowski (1968) has cast doubt on that. Trimble (1968) has compared the radial velocities and proper motions in the thick filamentary shell, which is a projected elliptical object with semiaxes of $3^{\prime}$ and $2^{\prime}$, and obtained a distance of $2 \mathrm{kpc}$ on the assumption that the 3-dimensional nebula is a prolate ellipsoid. Trimble and Woltjer (1971) have pointed out that the uncertainties in this value are large and that a distance as low as $1.2 \mathrm{kpc}$ or as high as $2.5 \mathrm{kpc}$ cannot be excluded.

Let us, however, be definite and adopt a distance of $2 \mathrm{kpc}$ for the Crab Nebula. This value seems to be the most widely used. Observations of the nebula indicate that energy must be supplied to it continuously. Assuming that the Crab pulsar is the only source of energy in the nebula, one can determine limits on the energy output of the pulsar (Rees and Trimble, 1970; Börner and Cohen, 1972) by considering the energy balance.

The only well established energy loss is by synchrotron radiation which implies $E_{\text {synch }}=1.2 \times 10^{38} \mathrm{erg} \mathrm{s}^{-1}$ (Baldwin, 1971), if the distance to the Crab is $2 \mathrm{kpc}$. The pulsar has to replenish at least the electrons producing the optical and X-ray synchrotron radiation because these particles have half-lives of less than $100 \mathrm{yr}$. So a rough estimate obtained from the observed spectrum (Baldwin, 1971) indicates that the pulsar has to supply continuously at least $0.8 \times 10^{38} \mathrm{erg} \mathrm{s}^{-1}$. For the pulsar to replenish this energy via loss of rotational energy its moment of inertia has to be at least $1.8 \times 10^{44} \mathrm{~g} \mathrm{~cm}^{2}$ (cf., Cohen and Cameron, 1971), corresponding to line (a) in Figure 7 of Paper I. Neutron star models corresponding to various equations of state have been discussed in that paper and it can be seen from Figure 2 that a model with $I=1.8 \times 10^{44} \mathrm{~g} \mathrm{~cm}^{2}$ has a mass of

$$
0.34 M_{\odot}(\mathrm{BPS}), \quad 0.36 M_{\odot}(\mathrm{BJ}), \quad 0.36 M_{\odot}(\mathrm{BBS}), \quad 0.26 M_{\odot}(\mathrm{CCLR}) .
$$

Here (BPS) corresponds to the equation of state published in Baym et al. (1971); (BJ) Bethe and Johnson (1973); (BBS) Bethe et al. (1970); (CCLR) Cohen et al. (1970). All the equations of state discussed in Paper I, except one, can easily provide neutron star models big enough to exceed this lower limit. The exception is the work of Leung and Wang (1971); the maximum moment of inertia in their equation of state, numbered (I) is less than $0.2 \times 10^{44} \mathrm{~g} \mathrm{~cm}^{2}$. That is, the Crab pulsar is definitely not 
among the stable neutron stars they compute using their equation of state which does not incorporate repulsion between baryons. Even Equation (II) of Leung and Wang (1971), which gives $I_{\max }=1.05 \times 10^{44} \mathrm{~g} \mathrm{~cm}^{2}$ is too low (Leung and Wang in Figure 7 of Paper I) although some repulsion is assumed to be present in this case (II). This indicates, as has been discussed already from the nuclear physics point of view by H. A. Bethe that the repulsion between the nucleons and hyperons at short distances plays an important role which may be the dominant feature at high densities. It is interesting to see that this is also suggested by astrophysical evidence.

The evidence in favor of repulsive interactions becomes even stronger if we take into account the protons that are pulled from the surface of the rotating neutron star by strong electric fields in the model proposed by Goldreich and Julian (1969). According to this model these protons are accelerated to the same energies as the electrons producing synchrotron radiation. In this case the minimum energy loss is twice the synchrotron radiation loss. This has the consequence that the minimum moment of inertia of the Crab increases by a factor of two:

$$
I_{\min } \geqslant 4 \times 10^{44} \mathrm{~g} \mathrm{~cm}^{2} \text {. }
$$

The neutron star mass is then $\geqslant 0.5 M_{\odot}$ (BBS, BJ). Thus if the model is valid we obtain the result that the Crab pulsar is a neutron star with a mass of at least $0.5 M_{\odot}$.

The acceleration mechanism of Gunn and Ostriker (1969) can also be tested. They require that the protons get ten times the energy of the electrons. Assuming the fluxes of electrons and jons from the pulsar to be equal, this would require ten times the synchrotron energy for the protons, leading to a moment of inertia of $2 \times 10^{45} \mathrm{~g} \mathrm{~cm}^{2}$ [line (c) in Figure 7 of Paper I]. Only the CCLR equation of state has models with moments of inertia of that magnitude. But even then this condition is satisfied only over a small density range near the mass peak. It therefore seems that the model of Gunn and Ostriker (1969) should be modified quantitatively.

Conclusions based on energy losses from the Crab nebula due to the expanding supernova shell (Rees and Trimble, 1970; Börner and Cohen, 1972) are much more uncertain than the preceding considerations. Observations of the filaments in the expanding supernova shell (Woltjer, 1958; Trimble, 1968) show that the expansion velocity at present is higher than would correspond to an expansion at constant velocity since 1054 . It seems that the nebula is accelerating now with an acceleration of

$$
\dot{v}=0.0014 \mathrm{~cm} \mathrm{~s}^{-2} \text {. }
$$

The nebula might, however, be decelerating now with the velocity still higher than the average if it had been accelerating rather strongly in the past. The energy of the expanding supernova shell would change due to this acceleration at a rate

$$
\dot{E}_{\mathrm{acc}} \doteq \int \varrho v \dot{v} d(\mathrm{Vol})
$$

where $\varrho$ is the density in the nebula. It would also change by the 'snow-plow' effect wherein the change in mass of the supernova shell as interstellar material piles up 
along the rim implies

$$
\dot{E}_{\text {plow }}=1 / 2 \varrho_{\mathrm{m}} v^{3} A,
$$

where $A$ is the surface area of the nebula and $\varrho_{m}$ is the density of interstellar material. Since we do not know whether the supernova shell is accelerating or decelerating at present, we investigate both cases. If the shell is decelerating, the energy gained by deceleration will be spent in the snowplow effect described by (2), and perhaps totally balance it. Thus $\dot{E}_{\text {shel1 }}=\dot{E}_{\text {acc }}+\dot{E}_{\text {plow }}=0$ is a distinct possibility. No further limits on the parameters of the Crab pulsar except those derived earlier from synchrotron radiation can be found in this case. We should notice, however, that in principle one could directly measure the value of $\dot{v}$ at present, and thus decide the question of acceleration or deceleration. If the currently accepted values for accelaration and snowplow are used, we find

$$
\begin{aligned}
\dot{E}_{\text {acc }} & =1.6 \times 10^{38} \mathrm{erg} \mathrm{s}^{-1}, \\
E_{\text {plow }} & =1.7 \times 10^{38} \mathrm{erg} \mathrm{s}^{-1}, \quad \text { and hence }, \\
E_{\text {shell }} & =3.3 \times 10^{3.8} \mathrm{erg} \mathrm{s}^{-1}
\end{aligned}
$$

This energy has to be supplied either directly by the pulsar via the low frequency waves emitted or by the adiabatic expansion of a relativistic gas (Trimble and Rees, 1970). In both cases a rotating neutron star with a moment of inertia of $10^{45} \mathrm{~g} \mathrm{~cm}^{2}$ can continuously supply that energy [line (b) in Figure 7 of Paper I]. The equations of state that can furnish a neutron star model with a moment of inertia big enough (BBS, BJ, CCLR) give a mass of $1.2 M_{\odot}$ (BBS, BJ) for this model. If little material was lost during the collapse the star would have had a mass of $1.35 M_{\odot}$ prior to the collapse - a mass above the Chandrasekhar limit for typical white dwarfs. It should be remembered that these energy losses are rather uncertain, and that the pulsar has to supply the energy continuously only if the energy content of the gas of relativistic particles in the nebula is maintained at its present level. All the uncertainties can, however, be decided by future observations and thus a value of $1.2 M_{\odot}$ for the Crab pulsar may be confirmed some day with a much higher degree of confidence than we have now.

If the shell is pushed out by low frequency waves from the pulsar and, if according to Gunn and Ostriker (1969), protons get ten times the energy of the electrons, then only the maximum mass (near the mass peak) neutron star models of the CCLR equation of state can fulfill this requirement, as indicated by line (d) in Figure 7 of Paper I. This particle acceleration mechanism therefore seems to be unrealistic.

If the distance of the Crab were less or more than $2 \mathrm{kpc}$, the limits derived above would have to be scaled down or up accordingly. If we go to the extreme values for the distance of $1.2 \mathrm{kpc}$ and $2.5 \mathrm{kpc}$, the moment of inertia necessary to account for the short-lived synchrotron particles would vary between 0.8 and $2.4 \times 10^{44} \mathrm{~g} \mathrm{~cm}^{2}\left[0.2 \mathrm{M}_{\odot}\right.$ and $0.4 M_{\odot}$ respectively (BBS)].

A final remark might be of interest. It pertains to the suggestion that cosmic rays 
are produced in the electromagnetic field of pulsars. The spectrum of galactic cosmic rays above $300 \mathrm{MeV}$ shows that about 50 times as much energy is present in protons as in electrons. It is clear from the foregoing discussion that the Crab pulsar could not produce such a ratio of proton to electron energy. This throws considerable doubt on the hypothesis that the high energy galactic cosmic rays are all produced by pulsars.

\section{The Vela Pulsar}

The Vela pulsar PSR 0833-45 is associated with the supernova remnant Vela X, which is about $1.1 \times 10^{4} \mathrm{yr}$ old, has a radius of $10 \mathrm{pc}$, and is at a distance of $500 \mathrm{pc}$ (Milne, 1970).

If the electromagnetic radiation emitted by Vela $X$ is synchrotron, then from synchrotron theory the energy content in the gas can be estimated at $\sim 10^{49}$ (Tucker, 1971). Assuming constant velocity, if the expansion velocity of the supernova shell were constant which would mean $v=880 \mathrm{~km} \mathrm{~s}^{-1}$, the energy loss through adiabatic expansion would be

$$
\dot{E}_{\text {ad }}=2.4 \times 10^{37} \mathrm{erg} \mathrm{s}^{-1} .
$$

On the other hand, the rotational energy lost by a neutron star with the parameters of PSR 0833-45 is between

$$
\dot{E}_{\text {rot }}=4 \times 10^{36} \mathrm{erg} \mathrm{s}^{-1} \text { to } 8.5 \times 10^{36} \mathrm{erg} \mathrm{s}^{-1}
$$

for models with mass between $0.8 M_{\odot}$ and $1.7 M_{\odot}$ (BBS). Although this is rather large compared to the loss of $4 \times 10^{35} \mathrm{erg} \mathrm{s}^{-1}$ in X-rays (Tucker, 1971) and $10^{33} \mathrm{erg} \mathrm{s}^{-1}$ in radio (Milne, 1970), the pulsar cannot supply the energy given in (6). The shell must therefore have been decelerating. Let us assume that the deceleration at present is very small, and that the main contribution to $E_{\text {shell }}$ is by snowplow (Börner and Cohen, 1972). If it is further assumed that a neutron star of $1.2 M_{\odot}$ is present to balance the expansion losses, then the velocity of expansion can be determined to be $240 \mathrm{~km} \mathrm{~s}^{-1}$ (Börner and Cohen, 1972). It is amusing to note that at about the same time Wallerstein and Silk (1971) independently (neither group knew of the other's work until after publication) measured the expansion velocity of Vela $\mathrm{X}$ by an observation of CaI lines in that direction, and they found precisely that value of $240 \mathrm{~km} \mathrm{~s}^{-1}$ for the expansion velocity.

\section{Pulsar Glitches}

The speed-ups of both Crab and Vela are discussed in this volume by D. Pines along with the various theoretical attempts to account for-them. The 'starquake' theory advocated by Pines in his paper, as well as the 'accretion' model (Börner and Cohen, 1972) are the only theories of pulsar glitches that derive limits on the mass (and other parameters) of Crab and Vela. Both theories use the two-component model for the interaction between the crust-charged particle system and the neutron superfluid to account for the relaxation phenomena of the post-speed-up behavior of the pulsar. 
They differ in the mechanism evoked to produce the initial glitch. Both theories are not without problems.

The starquake theory as described in Pine's paper views the smaller glitches of the Crab pulsar $\left(\Delta \Omega / \Omega \sim 10^{-9}\right)$ as a sudden relaxation of elastic stresses which accumulate in the crust (i.e., the rigid outer layers composed of a lattice of nuclei) of the pulsar as it is slowing down. The magnitude of this effect depends on the magnitude of the stresses that can be built up in the crust. Baym and Pines (1971) used an equation of state developed by Baym et al. (1972), where nuclei in the lattice become very large, up to $Z \sim 200$. On the other hand, J. Negele (in this volume) came to the conclusion that $Z \sim 40$ is to be expected in the crust of neutron stars. The amount of stress that can be built up increases monotonically with $Z$. It therefore seems that the crustquake theory has serious problems in explaining even the Crab pulsar glitches if the neutron star crust consists only of small nuclei. Even for $Z \sim 200$ nuclei in the lattice, the requirement to have a typical Crab speed-up every two years leads to a picture of the Crab pulsar as an almost completely solidified star. The crustquake theory predicts a mass of the Crab of less than $0.15 M_{\odot}$. This limit should not be taken too much at face value, but it illustrates the difficulties of this theory. A neutron star of $0.15 M_{\odot}$ might not even be formed in a supernova explosion (see Paper I) because its binding energy is so low that it becomes energetically more favorable to form dispersed ${ }^{56} \mathrm{Fe}$. Furthermore, $0.15 M_{\odot}$ disagrees with all the observational limits discussed above. The larger glitches of the Vela pulsar $\left(\Delta \Omega / \Omega \sim 10^{-6}\right)$ are ascribed to corequakes by Pines; that is, sudden relaxations of stress stored in the neutron star's central core made of a hadron lattice. The question of whether or not a solid hadron core may exist in neutron stars is discussed extensively in this volume, but no definite conclusion has been reached. The work of Canuto and Chitre (reported in these proceedings) suggests that a solid core might form at densities above $1.5 \times 10^{15} \mathrm{~g} \mathrm{~cm}^{-3}$. The corequake theory therefore predicts that the Vela pulsar has a central density of at least $1.5 \times 10^{15} \mathrm{~g} \mathrm{~cm}^{-3}$, which makes Vela a rather heavy neutron star with mass greater than $0.8 M_{\odot}$ (BPS), $1.2 M_{\odot}(\mathrm{BBS} ; \mathrm{CCLR})$, and $1.5 M_{\odot}$ (BJ) according to the various equations of state. The stress in the core is not built up between glitches in this model, but rather each glitch takes out a small part of a huge reservoir of elastic stress stored in the core. One has to explain why this produces glitches of $\left(\Delta \Omega / \Omega \sim 10^{-6}\right)$ instead of a continuous relaxation or one extremely big jump.

In the accretion model (Börner and Cohen, 1972) the initial glitch $(\Delta \Omega / \Omega)_{G}$ is ascribed to the infall of material onto the neutron star, transferring angular momentum to the crust and speeding it up. After some time the initial speed-up of the crust is transferred to the interior and the pulsar settles down to a long-term frequency increase $\Delta \Omega / \Omega$. If we write

$$
\left(\frac{\Delta \Omega}{\Omega}\right)_{G}=\frac{\Delta J_{\mathrm{c}}}{J_{\mathrm{c}}}-\frac{\Delta I_{\mathrm{c}}}{I_{\mathrm{c}}},
$$

with $J_{\mathrm{c}}$ as the angular momentum of the crust and $I_{\mathrm{c}}$ the moment of inertia of the crust, 
then

$$
\frac{\Delta \Omega}{\Omega}=\frac{\Delta J_{\mathrm{c}}}{J}-\frac{\Delta I}{I}
$$

By choosing a definite neutron star model, everything is determined from the measured quantities $(\Delta \Omega / \Omega)_{G}$ and $(\Delta \Omega / \Omega)$. Even the infalling mass $\Delta m$ can be found. In this simple model we find $\Delta m \sim 10^{-10} M_{\odot}$ for Crab glitches and $\Delta m \sim 10^{-6} M_{\odot}$ for Vela speed-ups. It is quite clear that initial conditions can be formulated which would exactly produce the observed behavior. A massive body flung off from the vicinity of the pulsar at some early stage in its life, not quite reaching escape velocity, but making just one loop and falling back onto a slowed down neutron star, would certainly transmit the right amount of angular momentum. It would also be able to accrete easily on the neutron star surface because it would have little excess angular momentum. The mass balance certainly is no problem, because $\Delta m$ is very small compared to typical pulsar masses. The big question is, however, whether the conditions prevailing after a supernova explosion can lead (with a certain non-zero probability) to the initial conditions needed for the accretion model. There certainly is a lot of homework to do, but not withstanding these theoretical difficulties we may point out that the accretion model agrees well with the observations and it also uses the same mechanism to explain the glitches of both Crab and Vela pulsars.

Viewing $\Delta \Omega / \Omega$ and $(\Delta \Omega / \Omega)_{G}$ as quantities determined (with a considerable uncertainty) by the observations, one derives from (8) and (9) the condition

$$
\sigma<\frac{\Delta I_{\mathrm{c}}-\Delta I}{I_{\mathrm{c}}} \frac{I}{I_{\mathrm{c}}} \frac{\Delta \Omega}{\Omega}-\left(\frac{\Delta \Omega}{\Omega}\right)_{\mathrm{G}},
$$

This leads to the condition that the mass of the Crab pulsar be greater than $1 M_{\odot}$; for Vela (10) does not impose any restrictions on the neutron star mass.

\section{Other Possibilities to Observe Neutron Stars}

\subsection{Pulsating X-RAY sources}

Recent observations from Uhuru have established the binary nature of two periodic pulsating X-ray sources: Cen X-3(Schreier et al., 1972) with a period of $4.84 \mathrm{~s}$ and Her X-1 (Tananbaum et al., 1972) with a period of $1.24 \mathrm{~s}$. A model may be suggested in which the $\mathrm{X}$-ray source is a neutron star emitting $\mathrm{X}$-rays by radiating as a black body in a number of hot spots. The rotation period of the neutron star provides the timing mechanism of the pulsation, and the X-rays are produced by the accretion of mass from a binary companion. Neutron stars accreting matter as models for X-ray sources have been proposed already by a number of authors (e.g., Shklovsky, 1967), but a reexamination of the older proposals in the light of these new observations might be worthwhile. We will not deal with the many intricate questions involved in such a model (cf., Borner et al. 1972), but just assume it to be valid and use the astronomical 
observation of the mass function $\left(M_{2}^{3} /\left(M_{1}+M_{2}\right)^{2}\right) \sin ^{3} i$ to get information on the mass of the neutron star. An optical identification of the main star will give its mass within rather narrow limits. The inclination $\sin i$ may be determined from an analysis of the pulse shape (Börner et al. (1972) find $\sin i \approx 1$ for Her X-1). Then the mass function will directly determine the mass $M_{1}$ of the neutron star. If, for example, for Her X-1, where $M^{3} /\left(M_{1}+M_{2}\right)^{2} \approx 0.85$ (for $\sin i \approx 1$ ), $M_{2}$ is an F-type star of $\sim 2 M_{\odot}$, then it follows that $M_{1} \approx 1 M_{\odot}$.

\subsection{GAMMA RAY LINES FROM OLD NEUTRON STARS}

Johnson et al. (1972) have reported low energy gamma ray observations from the galactic center region, showing a statistically significant spectral feature at $473 \pm 30 \mathrm{keV}$. This line emission with a total photon flux of $1.8 \times 10^{-3} \mathrm{~cm}^{-2} \mathrm{~s}^{-1}$ has been interpreted by Ramaty et al. (1972) as gravitationally redshifted positron annihilation radiation from the surface of old neutron stars. The production of positrons is attributed to nuclear reactions on the neutron star surface induced by the accretion of interstellar material. It is found that for an accretion rate of $10^{11} \mathrm{~g} \mathrm{~s}^{-1}$, or $6 \times 10^{34}$ particles s$^{-1}$, the redshifted positron annihilation yield of a single neutron star is $1.2 \times 10^{33}$ photons $\mathrm{s}^{-1}$. Thus, in order to account for the observations, a total of $1.5 \times 10^{10}$ old neutron stars in the galactic center region is required. Positron annihilation radiation is normally at $511 \mathrm{keV}$, so this interpretation requires redshifts ranging from 0.016 to 0.13 . Since a given redshift completely determines a specific neutron star model, as a consequence the majority of these old neutron stars have masses of less than $0.8 M_{\odot}$. While the numbers are probably quite uncertain still, there appears the interesting possibility that observations of redshifted gamma ray lines provide a direct measurement of the general distribution of the physical parameters of neutron stars. The principal observational tests of the model of Ramaty et al. (1972) would be the detection of nuclear gamma ray lines from the galactic center and redshifted positron annihilation radiation from the galactic disk.

An accretion rate of $10^{17} \mathrm{~g} \mathrm{~s}^{-1}$ (assuming a surface composition of CNO or heavier nuclei) should produce about $10^{39}$ positrons $\mathrm{s}^{-1}$ from a single neutron star, and (if the object is at a distance of $3 \mathrm{kpc}$ ) a flux of redshifted positron annihilation radiation at Earth of $10^{-6}$ protons $\mathrm{cm}^{-2} \mathrm{~s}^{-1}$. This gamma ray flux is below the presently available detector sensitivities, but in the future we might perhaps be able to see the redshifted positron annihilation radiation from binary X-ray sources or from single nearby neutron stars.

\section{References}

Baldwin, J. E.: 1971, in R. D. Davies and F. G. Smith (eds.), 'The Crab Nebula', IAU Symp. 46, 22. Baym, G. and Pines, D.: 1971, A. de Shalit Memorial Volume.

Baym, G., Bethe, H. A., and Pethick, C.: 1972, (to be published).

Baym, G., Pethick, C., and Sutherland, P.: 1971, Astrophys. J. 179, 200.

Bethe, H. A. and Johnson, M.: 1973 (to be published).

Bethe, H. A., Börner, G., and Sato, K.: 1970, Astron. Astrophys. 7, 279.

Biermann, L.: 1972, Boulder Lecture ('Cosmic Plasma Physics').

Börner, G. and Cohen; J. M.: 1972, Astron. Astrophys. 19, 109. 
Börner, G., Meyer, F., Schmidt, H. U., and Thomas, H. C.: 1972, Contribution to Fall Meeting of the DAG, Wien.

Cohen, J. M. and Cameron, A. G. W.: 1971, Astrophys. Space Sci. 10, 227.

Cohen, J. M., Langer, W. D., Rosen, L. C., and Cameron, A. G. W.: 1970, Astrophys. Space Sci. 6, 228.

Goldreich, P. and Julian, W. H.: 1969, Astrophys. J. 157, 869.

Gunn, J. E. and Ostriker, J. P.: 1969, Astrophys. J. 157, 1395.

Johnson III, W. N., Harnden, F. R., and Haymes, R. C.: 1972, Astrophys. J. Letters 172, L1.

Leung, Y. C. and Wang, C. G.: 1971, Astrophys. J. 170, 499.

Manchester, R. N. and Taylor, J. G.: 1972, Astrophys. Letters 10, 67.

Maran, S. P. and Cameron, A. G. W.: 1969, Earth Extraterrestr. Sci. 1, 3.

Milne, D. K.: 1970, Australian J. Phys. 23, 425.

Minkowski, R.: 1968, Stars and Stellar Systems 7, Ch. 11.

Ramaty, R., Börner, G., and Cohen, J. M.: 1972 (to be published).

Schreier, E., Levinson, R., Gursky, H., Kellogg, E., Tananbaum, H., and Giacconi, R.: 1972, Astrophys. J. Letters 172, L79.

Shklovsky, I.: 1967, Astrophys. J. Letters 150, L48.

Tananbaum, H., Gursky, H., Kellogg, E. M., Levinson, E., Schreier, E., and Giacconi, R.: 1972, Astrophys. J. Letters 174, L143.

Trimble, V.: 1968, Astron. J. 73, 535.

Trimble, V. and Rees, M. J.: 1970, Astrophys. Letters 5, 93.

Trimble, V. and Woltjer, L.: 1971, Astrophys. J. Letters 163, L97.

Tucker, W. H.: 1971, Astrophys. J. Letters 167, L85.

Wallerstein, G. and Silk, J.: 1971, Astrophys. J. 170, 289.

Woltjer, L.: 1958, Bull. Astron. Inst. Neth. 14, 39. 